\title{
Fractional Generalization of Fick's Law: A Microscopic Approach
}

\author{
I. Calvo, ${ }^{1}$ R. Sánchez, ${ }^{2}$ B. A. Carreras, ${ }^{3}$ and B. Ph. van Milligen ${ }^{1}$ \\ ${ }^{1}$ Laboratorio Nacional de Fusión, Asociación EURATOM-CIEMAT, 28040 Madrid, Spain \\ ${ }^{2}$ Fusion Energy Division, Oak Ridge National Laboratory, Oak Ridge, Tennessee 37831, USA \\ ${ }^{3}$ BACV Solutions Inc., Oak Ridge, Tennessee 37830, USA
}

(Received 26 July 2007; published 7 December 2007)

\begin{abstract}
In the study of transport in inhomogeneous systems it is common to construct transport equations invoking the inhomogeneous Fick law. The validity of this approach requires that at least two ingredients be present in the system. First, finite characteristic length and time scales associated with the dominant transport process must exist. Second, the transport mechanism must satisfy a microscopic symmetry: global reversibility. Global reversibility is often satisfied in nature. However, many complex systems exhibit a lack of finite characteristic scales. In this Letter we show how to construct a generalization of the inhomogeneous Fick law that does not require the existence of characteristic scales while still satisfying global reversibility.
\end{abstract}

DOI: 10.1103/PhysRevLett.99.230603

PACS numbers: 05.60.- $\mathrm{k}, 05.10 . \mathrm{Gg}, 05.40 . \mathrm{Fb}$

Over a hundred years ago, Fick proposed the famous formula that bears his name while studying transport processes in saline aqueous solutions [1]. He observed that a salt flux was driven by the presence of a salt density gradient (in the absence of other forces) and hypothesized that they were linearly related to each other. In one dimension, Fick's relation would read

$$
\Gamma_{F}=-A \frac{\partial n}{\partial x}
$$

where $n$ is the salt density and the minus sign makes sure that the flux tends to reduce the gradient. Since then, Fick's law has found application in countless systems, and expressions like Eq. (1) are applied to the transport of many quantities: electrons and ions, neutral particles, energy or momentum, risk, chemical reactants, or currency, just to name a few.

Using Fick's expression in the standard continuity equation yields the familiar classical diffusive equation,

$$
\frac{\partial n}{\partial t}=A \frac{\partial^{2} n}{\partial x^{2}}
$$

where $n$ represents now the density of the quantity of interest. The constant $A$ is the diffusive coefficient, which is related to some well-defined characteristic length $(l)$ and time $(\tau)$ scales of the underlying dominant transport process (in the case of salt transport, the mean-free-path between collisions with the background water molecules and the intercollision time): $A \sim l^{2} / \tau$. By well-defined, we mean that $l$ and $\tau$ are both finite and much smaller than the system size and lifespan, respectively.

Many physical systems are, however, inhomogeneous. The usual inhomogeneous extension of Fick's law is

$$
\Gamma_{F}=-A(x) \frac{\partial n}{\partial x} \Rightarrow \frac{\partial n}{\partial t}=\frac{\partial}{\partial x}\left[A(x) \frac{\partial n}{\partial x}\right] .
$$

In spite of its familiarity, this expression is not the only possible extension that reduces to Eq. (2) when $A(x)=$ $A \equiv$ const. In fact, Fick's law is a particular case of the more general Fokker-Planck diffusive law [2], that defines the flux by,

$$
\Gamma_{\mathrm{FP}}=B(x) n-\frac{\partial}{\partial x}[A(x) n] .
$$

Equation (3) requires that $B(x)=d A(x) / d x$. Traditionally, the validity of this constraint [and thus Eq. (3)] has been justified by invoking the presence of a particular symmetry of the underlying microscopic transport mechanism: local reversibility (LR) [2]. The symmetry applies when the probability (per unit time) of a particle being transported from any location $x$ to any other location $x^{\prime}$ equals that of being transported from $x^{\prime}$ to $x$ [for instance, consider reactants moving in a reactive medium. LR holds whenever the probability rate of moving between any $x$ and $x^{\prime}$ without reacting depends on the total number of reactive centers found along the reactant path. Similar situations are very common in nature]. The condition for the validity of Fick's law is, however, less stringent than LR: global reversibility (GR) is sufficient. Namely, that the probability (per unit time) of a particle leaving $x$ be the same as the probability (per unit time) of arriving at $x$. Clearly, LR implies GR but the converse is not true [2].

The purpose of this Letter is to revisit this discussion in a different but related context, which has emerged as an important paradigm in the last decade: that of scale-free (or fractional) diffusion [3,4]. Scale-free transport appears in systems in which some of the transport characteristic scales, on which Fick's law is fundamentally based, are absent. The question we will try to answer in this Letter is: which is the expression that correctly represents fluxes in an inhomogeneous system satisfying GR but lacking such characteristic scales? The relevance and timeliness of the answer is justified by the recent surge of interest in physical, economical, biological and social systems in which 
scale-free transport is observed [3,4]. An example is provided by those systems in which (particle or energy) transport takes place via correlated avalanches. This seems to be the case of some magnetically confined fusion plasmas [57], the propagation of forest fires [8], earthquakes [9] or solar flares [10]. Transport events in these cases have a maximum size that is only limited by the system size $L$, and therefore a characteristic size that diverges with (some power of) $L$. In some of these systems, LR is satisfied at least in certain limits. For instance, in the propagation of forest fires, the transition rate at which fire propagates from tree A to tree B would heavily depend on how many active trees exist between them, thus satisfying LR.

The discussion will proceed in parallel to the previous one on classical diffusion. We start by reviewing the scalefree version of the homogeneous Fick law [3,4]. The relevant transport equation is then expressed in terms of fractional differential operators (FDOs) [11]. To understand why, we consider the simplest one, known as the Markovian, symmetric fractional diffusion equation (MsFDE):

$$
\frac{\partial n}{\partial t}=A_{\alpha} \frac{\partial^{\alpha} n}{\partial|x|^{\alpha}}, \quad \alpha \in(0,2],
$$

where $A_{\alpha}$ is a constant. The Riesz FDO is defined as

$$
\frac{\partial^{\alpha}}{\partial|x|^{\alpha}} \equiv \frac{-1}{2 \cos (\pi \alpha / 2)}\left({ }_{-\infty} D_{x}^{\alpha}+{ }^{\infty} D_{x}^{\alpha}\right),
$$

where the Riemann-Liouville FDOs of order $\alpha$ are [11]

$$
\begin{aligned}
& a D_{x}^{\alpha} f \equiv \frac{1}{\Gamma(m-\alpha)} \frac{d^{m}}{d x^{m}} \int_{a}^{x} \frac{f\left(x^{\prime}\right)}{\left(x-x^{\prime}\right)^{\alpha-m+1}} d x^{\prime}, \\
& a D_{x}^{\alpha} f \equiv \frac{(-1)^{m}}{\Gamma(m-\alpha)} \frac{d^{m}}{d x^{m}} \int_{x}^{a} \frac{f\left(x^{\prime}\right)}{\left(x^{\prime}-x\right)^{\alpha-m+1}} d x^{\prime},
\end{aligned}
$$

being $m$ the integer number verifying that $m-1 \leq \alpha<$ $m$. In spite of their complicated appearance, the theory of fractional differential operators is very well-established [11]. The feature of FDOs which makes them relevant to the context of scale-free transport is their action in Fourier space

$$
\mathcal{F}\left[{ }_{-\infty} D_{x}^{\alpha} f\right]=(-i k)^{\alpha} \hat{f}, \quad \mathcal{F}\left[{ }^{\infty} D_{x}^{\alpha} f\right]=(i k)^{\alpha} \hat{f},
$$

where $\hat{f}(k) \equiv \mathcal{F}[f](k)$. Using property (8) it follows that the Riesz FDO satisfies

$$
\mathcal{F}\left[\frac{\partial^{\alpha} f}{\partial|x|^{\alpha}}\right](k)=-|k|^{\alpha} \hat{f}(k) .
$$

This result justifies the name "fractional differential operators", since they naturally extend the notion of derivative to noninteger order. What is most relevant to us is that they also provide a suitable generalization of classical diffusion. Taking the Fourier transform of Eq. (5), we obtain

$$
\frac{\partial \hat{n}(k)}{\partial t}=-A_{\alpha}|k|^{\alpha} \hat{n}(k),
$$

which reduces to the classical diffusive equation [Eq. (2)] for $\alpha=2$ (note that for $\alpha=2$ the Riesz FDO yields the second derivative operator). This fractional extension is relevant for systems in which transport characteristic scales diverge. To illustrate this point, let us examine the propagator [i.e., the temporal evolution of an initial condition $\left.n(x, 0)=\delta\left(x-x_{0}\right)\right]$ of Eq. (5). The time derivative of the second moment of the propagator is easily related to the transport characteristic length scale $l$. In the case of the diffusive equation $(\alpha=2)$ the propagator is a Gaussian function,

$$
G\left(x, t \mid x_{0}, 0\right)=(4 \pi A t)^{-1 / 2} \exp \left\{-\left(x-x_{0}\right)^{2} /(4 A t)\right\},
$$

which has a finite second moment that increases linearly with time and thus yields a finite $l$. However, for $\alpha<2$, the propagator is given by a symmetric Lévy distribution of order $\alpha$ and behaves like $\left(\left|x-x_{0}\right| \gg 1\right)$ :

$$
G\left(x, t \mid x_{0}, 0\right) \sim C_{\alpha} t^{-1 / \alpha}\left(\frac{\left|x-x_{0}\right|}{t^{1 / \alpha}}\right)^{-(1+\alpha)},
$$

where $C_{\alpha}$ is a constant [12]. Because of its fat power-law tail, the second moment of this propagator is infinite. Thus, the transport described by this equation lacks a finite characteristic length. This result, together with the fact that Lévy distributions are stable with respect to the central limit theorem (as the Gaussian law) [12], endows FDOs with the physical basis needed to justify their use to model scale-free transport. Let us define, for convenience, the operator

$$
a F_{x}^{\beta} \equiv \frac{-1}{2 \cos [\pi(\beta+1) / 2]}\left({ }_{-a} D_{x}^{\beta}-{ }^{a} D_{x}^{\beta}\right) .
$$

Then, the fractional particle flux associated to Eq. (5) can be written as

$$
\Gamma_{F ; \alpha}=-A_{\alpha}\left({ }_{\infty} F_{x}^{\alpha-1} n\right) .
$$

Evidently, the operator $a F_{x}^{\beta}$ satisfies that

$$
\frac{\partial}{\partial x}\left({ }_{\infty} F_{x}^{\alpha-1}\right)=\frac{\partial^{\alpha}}{\partial|x|^{\alpha}} .
$$

Equation (13) is the fractional generalization of the homogeneous Fick law, Eq. (1), which is recovered for $\alpha=2$ due to the fact that $\infty F_{x}^{1}=d / d x$.

We now come to derive the central result of this Letter: the inhomogeneous extension of (5) that preserves GR or, in other words, the scale-free (fractional) generalization of the inhomogeneous Fick law, Eq. (3). We start by giving precise expressions for the GR/LR symmetries and then we present a new way to derive the inhomogeneous Fick law (Eq. (3)). The need for a new approach is caused by the failure of the standard methods to derive the inhomogeneous Fick law when characteristic length scales are lack- 
ing. Finally, we show that the new procedure works in the fractional case, yielding the fractional generalization of the inhomogeneous Fick law. For the sake of simplicity, we restrict ourselves to the discussion of the Markovian case, but the extension to include non-Markovianity is rather straightforward.

The continuous-time random walk (CTRW) [13] provides a suitable framework to derive macroscopic transport equations from the microscoscopic dynamics. CTRWs are models describing a large number of particles whose motion is defined probabilistically using a probability density function (PDF). For our purposes, it is enough to consider one-dimensional separable models defined by two PDFs: a step-size $\mathrm{PDF}, p\left(\Delta_{x} ; x\right)$, and a waiting-time $\mathrm{PDF}, \psi\left(\Delta_{t} ; x\right)$, $\Delta_{t} \geq 0$. The joint PDF $\xi\left(\Delta_{x}, \Delta_{t} ; x\right)=p\left(\Delta_{x} ; x\right) \psi\left(\Delta_{t} ; x\right)$ gives the probability that a particle located at $x$ at time $t$ jumps to $x+\Delta_{x}$ at time $t+\Delta_{t}$. As mentioned above, we restrict the discussion to Markovian problems by selecting a Poisson law for the waiting-time PDF: $\psi\left(\Delta_{t} ; x\right)=$ $\tau(x)^{-1} \exp \left(-\Delta_{t} / \tau(x)\right)$, where $\tau(x)$ is the mean waiting time at $x$. Imposing conservation of the number of particles leads $[14,15]$ to the generalized master equation (GME) governing the time evolution of the density of particles $n(x, t)$ :

$$
\frac{\partial n(x, t)}{\partial t}=\int_{-\infty}^{\infty} \frac{n\left(x^{\prime}, t\right)}{\tau\left(x^{\prime}\right)} p\left(x-x^{\prime} ; x^{\prime}\right) d x^{\prime}-\frac{n(x, t)}{\tau(x)} .
$$

The GME is the starting point in the derivation of (macroscopic) fluid transport equations, by going to the fluid limit in which only long-time, large-distance information is retained. This can be easily done in Fourier space by taking the limit of small $k$. The Fourier transform of the GME (15) with respect to $x$ yields [16]:

$$
\frac{\partial \hat{n}(k, t)}{\partial t}=\int_{-\infty}^{\infty} \frac{n\left(x^{\prime}, t\right)}{\tau\left(x^{\prime}\right)}\left[\hat{p}\left(k ; x^{\prime}\right)-1\right] e^{i k x^{\prime}} d x^{\prime},
$$

where $\hat{p}\left(k ; x^{\prime}\right)=\int_{-\infty}^{\infty} p\left(\Delta ; x^{\prime}\right) e^{i k \Delta} d \Delta$ is the characteristic function of $p\left(\Delta ; x^{\prime}\right)$. We introduce now the characteristic exponent, $\Lambda\left(k ; x^{\prime}\right)$, through $\hat{p}\left(k ; x^{\prime}\right) \equiv \exp \Lambda\left(k ; x^{\prime}\right)$. The fluid limit of the GME is obtained then by performing the small $k$ approximation $\hat{p}\left(k ; x^{\prime}\right) \approx 1+\Lambda\left(k ; x^{\prime}\right)$, which turns Eq. (16) into

$$
\frac{\partial \hat{n}(k, t)}{\partial t}=\int_{-\infty}^{\infty} \frac{n\left(x^{\prime}, t\right)}{\tau\left(x^{\prime}\right)} \Lambda\left(k ; x^{\prime}\right) e^{i k x^{\prime}} d x^{\prime} .
$$

First, let us show how to obtain the homogeneous classical [Eq. (2)] and fractional [Eq. (5)] diffusive equations from Eq. (17). We assume that both $\tau\left(x^{\prime}\right)$ and $\Lambda\left(k ; x^{\prime}\right)$ are independent of $x^{\prime} . \tau$ becomes then the characteristic transport time scale. The characteristic length scale is related (if it exists) to the variance $\sigma_{2}$ of the step-size PDF, $p(\Delta)$. In the absence of external forces, the central limit theorem guides us to choose either a Gaussian or a symmetric Lévy distribution for $p(\Delta)$ [12]. The characteristic exponent of the Gaussian is $\Lambda(k)=-\sigma_{2} k^{2}$, which inserted into
Eq. (17) yields the (Fourier transform of the) classical diffusive Eq. (2) with $A=\sigma_{2} / \tau$. The finite variance of the Gaussian is related to a finite characteristic length scale $l \sim \sqrt{\sigma_{2}}$. In the case of a symmetric Lévy PDF, the characteristic exponent is [12] $\Lambda(k)=-\sigma_{\alpha}|k|^{\alpha}$, with $0<\alpha<$ 2. The fluid limit of Eq. (17) then reduces to (the Fourier transform of) Eq. (5). The infinite variance of the Lévy PDFs implies that $l$ diverges.

We will now formalize the microscopic symmetries underlying the inhomogeneous Fick law [Eq. (3)] that we discussed briefly at the beginning of this Letter. First, we introduce the 1-particle transition rate, $T(\Delta ; x)=$ $p(\Delta ; x) / \tau(x)$. The LR condition is then expressed as [2] $T(-\Delta, x+\Delta)=T(\Delta, x), \forall x$. As mentioned, Fick's law only requires the less stringent GR condition,

$$
\int_{-\infty}^{\infty} T(-\Delta, x+\Delta) d \Delta=\int_{-\infty}^{\infty} T(\Delta, x) d \Delta, \quad \forall x,
$$

as will be shown in the following.

To derive the inhomogeneous Fick law in the classical (Gaussian) case, one would typically enforce the symmetry [Eq. (18)] after Taylor expanding the right-hand side of Eq. (15) around $x$ and keeping only the terms involving up to the second moment of $T$. However, a Lévy PDF of order $\alpha<2$ does not have finite moments of order equal to or greater than $\alpha$. Hence, we need to develop a procedure to find the fluid limit equations while imposing GR which does not require an expansion in moments. The key point is that, after some straightforward manipulations, the symmetry (18) can be recast into the following, equivalent form:

$$
\int_{-\infty}^{\infty}\left[\frac{\hat{p}\left(k, x^{\prime}\right)-1}{\tau\left(x^{\prime}\right)}\right] e^{i k x^{\prime}} d x^{\prime}=0, \quad \forall k .
$$

The fluid limit of the symmetry can be expressed in terms of the characteristic exponent:

$$
\int_{-\infty}^{\infty} \frac{\Lambda\left(k, x^{\prime}\right)}{\tau\left(x^{\prime}\right)} e^{i k x^{\prime}} d x^{\prime}=0,
$$

which is the small $k$ approximation of Eq. (19).

It is instructive to rederive the ordinary, inhomogeneous Fick law, Eq. (3), using this formalism. Consider the most general form of the characteristic exponent of the Gaussian law allowed by the central limit theorem [12]:

$$
\Lambda\left(k ; x^{\prime}\right)=i a\left(x^{\prime}\right) k-\sigma_{2}\left(x^{\prime}\right) k^{2} .
$$

Inserting this expression into (20) we immediately find that the following relation between $\bar{a}\left(x^{\prime}\right)=a\left(x^{\prime}\right) / \tau\left(x^{\prime}\right)$ and $\bar{\sigma}_{2}\left(x^{\prime}\right)=\sigma_{2}\left(x^{\prime}\right) / \tau\left(x^{\prime}\right)$ must hold

$$
\hat{\bar{a}}(k)=-i k \hat{\bar{\sigma}}_{2}(k),
$$

or, in real space

$$
\bar{a}\left(x^{\prime}\right)=\frac{d \bar{\sigma}_{2}\left(x^{\prime}\right)}{d x^{\prime}} .
$$


This relation will be recognized as the one invoked earlier to reduce the full Fokker-Planck law to the inhomogeneous Fick law. Using Eqs. (21) and (23) and Fourier inverting Eq. (17), the classical inhomogeneous Fick law is recovered:

$$
\frac{\partial n}{\partial t}=-\frac{\partial \Gamma_{F}}{\partial x}, \quad \Gamma_{F}=-\bar{\sigma}_{2}(x) \frac{\partial n}{\partial x} .
$$

We can use this very same scheme to derive the fractional version of the inhomogeneous Fick law in the setup of Lévy PDFs with algebraic tails that are stable according to the central limit theorem [12]. The relevant fractional generalization of Eq. (21) is now

$$
\Lambda\left(k ; x^{\prime}\right)=i a\left(x^{\prime}\right) k-\sigma_{\alpha}\left(x^{\prime}\right)|k|^{\alpha}, \quad \alpha \in(0,2),
$$

with $\sigma_{\alpha}\left(x^{\prime}\right)>0$. Imposing that Eq. (25) satisfy again GR (Eq. (20)) yields $i k \hat{\bar{a}}(k)-|k|^{\alpha} \hat{\bar{\sigma}}_{\alpha}(k)=0$, where $\bar{a}\left(x^{\prime}\right)=$ $a\left(x^{\prime}\right) / \tau\left(x^{\prime}\right), \bar{\sigma}_{\alpha}\left(x^{\prime}\right)=\sigma_{\alpha}\left(x^{\prime}\right) / \tau\left(x^{\prime}\right)$. The fractional version of Eq. (22) thus becomes

$$
\hat{\bar{a}}(k)=\frac{|k|^{\alpha}}{i k} \hat{\bar{\sigma}}_{\alpha}(k) .
$$

Using the identity $2 \cos (\pi \alpha / 2)|k|^{\alpha}=(i k)^{\alpha}+(-i k)^{\alpha}$ we can rewrite Eq. (26) as

$$
\hat{\bar{a}}(k)=\frac{(i k)^{\alpha-1}-(-i k)^{\alpha-1}}{2 \cos (\pi \alpha / 2)} \hat{\bar{\sigma}}_{\alpha}(k),
$$

and making use of the properties of FDOs under Fourier transforms discussed previously:

$$
\bar{a}\left(x^{\prime}\right) \underset{\infty}{=} F_{x^{\prime}}^{\alpha-1} \bar{\sigma}_{\alpha},
$$

where the operator $\infty F_{x}^{\alpha-1}$ was defined in Eq. (12). Equation (28) is the fractional generalization of the classical relation (23). Using this result and Eq. (25) in Eq. (17), and Fourier inverting, we obtain the sought-for fractional generalization of the inhomogeneous Fick law, $\partial_{t} n=-\partial_{x} \Gamma_{F ; \alpha}$, where the fractional Fick flux is now given by

$$
\Gamma_{F ; \alpha}=-\left[{ }_{\infty} F_{x}^{\alpha-1}\left(\bar{\sigma}_{\alpha} n\right)-\left({ }_{\infty} F_{x}^{\alpha-1} \bar{\sigma}_{\alpha}\right) n\right] .
$$

Equation (29) is the central result of this Letter. It provides the keystone on which any description of transport in systems lacking characteristic length scales but still satisfying GR (or LR) should be built. As we mentioned, an example could be provided by the propagation of forest fires across an inhomogeneous distribution of trees. Another possible application might be the modeling of some aspects of radial particle transport in turbulent fusion plasmas, such as those confined in certain tokamak regimes $[17,18]$. Additional terms might be needed in this case to account for additional effects associated to radially varying temperatures and external forcing. Note also that Eq. (29) is very different from the extension that one would expect from Eq. (13) in the spirit of Eq. (3). Namely,

$$
\Gamma_{F ; \alpha} \neq-\bar{\sigma}_{\alpha}(x)\left({ }_{\infty} F_{x}^{\alpha-1} n\right) .
$$

The reason is that fractional derivatives do not satisfy the Leibniz rule for the derivative of the product of two functions [11]. Hence, Eq. (30) is an equality only in the special case $\alpha=2$. Needless to say, other fractional generalizations [including the one where the flux is defined by the right-hand side of Eq. (30)] might also be valid under different assumptions.

Finally, we would like to point out that the scheme laid out in this Letter also provides the basis for addressing the derivation of the fractional Fick law in three-dimensional inhomogeneous systems. The CTRW construction can be trivially extended to any dimension. However, the formulation in terms of fractional derivative operators, even in cases in which rotational invariance reduces the problem to one effective dimension, requires tackling a number of nontrivial technical details, which we plan to study in a future work.

Part of this research was sponsored by the Laboratory Research and Development Program of the Oak Ridge National Laboratory, managed by UT-Battelle, LLC, for the US-DOE under Contract No. DE-AC05-00OR22725.

[1] A. Fick, Ann. Phys. (Leipzig) 170, 59 (1855).

[2] N.G. van Kampen, Stochastic Processes in Physics and Chemistry (North Holland, New York, 1981).

[3] R. Metzler and J. Klafter, Phys. Rep. 339, 1 (2000).

[4] G. Zaslavsky, Phys. Rep. 371, 461 (2002).

[5] D. E. Newman et al., Phys. Plasmas 3, 1858 (1996).

[6] B. A. Carreras et al., Phys. Plasmas 3, 2903 (1996).

[7] R. Sanchez et al., Nucl. Fusion 41, 247 (2001).

[8] B. Drossel et al., Phys. Rev. Lett. 69, 1629 (1992).

[9] B. E. Shaw et al., J. Geophys. Res. 97, 479 (1992).

[10] E. Lu and R. J. Hamilton, Astrophys. J. 380, L89 (1991).

[11] I. Podlubny, Fractional Differential Equations (Academic Press, New York, 1998).

[12] G. Samorodnitsky and M. S. Taqqu, Stable Non-Gaussian Processes (Chapman \& Hall, New York, 1994).

[13] E. W. Montroll and G. Weiss, J. Math. Phys. (N.Y.) 6, 167 (1965).

[14] V.M. Krenke, E.W. Montroll, and M.F. Schlesinger, J. Stat. Phys. 9, 45 (1973).

[15] B. P. van Milligen, R. Sanchez, and B. A. Carreras, Phys. Plasmas 11, 2272 (2004).

[16] B. P. van Milligen, B. A. Carreras, and R. Sanchez, Plasma Phys. Controlled Fusion 47, B743 (2005).

[17] B. A. Carreras et al., Phys. Plasmas 8, 5096 (2001).

[18] D. del-Castillo-Negrete et al., Phys. Plasmas 11, 3854 (2004). 